\title{
Electric Field Effect Tuning of Electron-Phonon Coupling in Graphene
}

\author{
Jun Yan, ${ }^{1}$ Yuanbo Zhang, ${ }^{1}$ Philip Kim, ${ }^{1}$ and Aron Pinczuk ${ }^{1,2}$ \\ 1 Department of Physics, Columbia University, New York, NY 10027, USA \\ 2 Department of Applied Physics and Applied Mathematics, \\ Columbia University, New York, NY 10027, USA
}

(Dated: October 29, 2018)

\begin{abstract}
Gate-modulated low-temperature Raman spectra reveal that the electric field effect (EFE), pervasive in contemporary electronics, has marked impacts on long wavelength optical phonons of graphene. The EFE in this two dimensional honeycomb lattice of carbon atoms creates large density modulations of carriers with linear dispersion (known as Dirac fermions). Our EFE Raman spectra display the interactions of lattice vibrations with these unusual carriers. The changes of phonon frequency and line-width demonstrate optically the particle-hole symmetry about the charge-neutral Dirac-point. The linear dependence of the phonon frequency on the EFE-modulated Fermi energy is explained as the electron-phonon coupling of mass-less Dirac fermions.
\end{abstract}

The interaction between electrons and quantized lattice vibrations in a solid is one of the most fundamental realms of study in condensed matter physics. In particular, the electron-phonon interaction in graphene and its derivatives plays an important role in understanding anomalies of photoemission spectra observed in graphite [1] and graphene 2], the non-linear high energy electron transport in carbon nanotubes [3, 4, 5, 6, 7], as well as phonon structures in graphite [8, 9] and carbon nanotubes [9, 10, 11].

Traditionally, electron-phonon interactions are investigated through chemical doping, in which the charge carrier density is varied by introduction of impurities. The electric field effect (EFE) is an alternative method for changing the charge carrier density effectively in lowdimensional systems. The EFE has proven very successful in graphene, a single atomic sheet of graphite, where unconventional integer quantum Hall effect [12, 13] has revealed physics linked to the uniqueness of the electronic band structure near the charge neutral Dirac points (Fig. 1(a)).

We measured Raman spectra of optical phonons in graphene where large densities of free electrons or free holes are modulated by the EFE. We discovered that the even parity long wavelength optical phonon (the graphene $\mathrm{G}$ band) has marked dependence on gate voltage and the induced charge density. The dependence of phonon frequency and line-width on the EFE induced charge density demonstrates that the intriguing physics of mass-less Dirac fermions with particle-hole symmetry is encoded in the electron-phonon interaction.

Raman studies of graphite [14] are at the forefront of research on carbon based materials. The recent availability of few-layer and single-layer graphene [15, 16], has stimulated great interest in Raman scattering in such novel and exciting systems. For example, dimensional crossover was observed in Raman spectra of thin graphitic films as a function of multilayer thickness [17, 18, 19]. In the work reported here, Raman spectroscopy emerges as an insightful method to probe the
EFE in a single atomic layer and the phonon dynamics that are associated with the two dimensional (2D) Dirac fermions.

We focus on the doubly degenerate optical phonon of $\mathrm{E}_{2 g}$ symmetry at $\sim 1580 \mathrm{~cm}^{-1}$, known as the $\mathrm{G}$ band. We also report on the smaller impact of the EFE on the second-order band at $\sim 2700 \mathrm{~cm}^{-1}$, known as the $\mathrm{D}^{*}$ band. These two bands are prominent Raman features in graphene [17, 18, 19]. G band is the optical phonon at long wavelengths and $\mathrm{D}^{*}$ band is associated with a twophonon state in which each phonon has a large wavevector. Our experiments demonstrate that the $\mathrm{G}$ band is markedly sensitive to coupling with Dirac fermion excitations at small wavevectors (long wavelengths), while the reduced impact of the EFE on the $\mathrm{D}^{*}$ band offers insights on the coupling to particle-hole pairs at large wavevectors.

The interaction of the $\mathrm{G}$ phonon with small wavevector particle-hole pairs has a crucial dependence on the onset energy $\varepsilon_{c}$ for vertical transitions (zero momentum transfer) of an electron from $\pi$ valence band to a $\pi^{*}$ conduction band state as shown in Figs. 1(b) and 1(c). The $\pi \rightarrow \pi^{*}$ transitions need to satisfy the requirements of the Pauli principle. For this reason, $\varepsilon_{c}$ is directly linked to the position of the Fermi surface as $\varepsilon_{c}=2\left|E_{F}\right|$, where $\left|E_{F}\right|$ is the absolute value of the Fermi energy. The response of the G phonon to the EFE is thus inextricably related to the electronic band structure in the vicinity of the Dirac point.

Graphene samples were prepared using the mechanical method described in Ref. [20]. The sample was mounted on degenerately doped p-type silicon with a 300-nm thick $\mathrm{SiO}_{2}$ as gate dielectric. A gold electrode was thermally evaporated on one end of the graphene layer to contact it to the electrical ground (Fig. 1(d)). A gate bias voltage $V_{g}$ was applied to the silicon substrate to induce charge carriers in the sample by the EFE. The sample was mounted in a variable temperature cryostat with optical access where it was cooled by a continuous flow of cold Helium gas. The Raman measurements were performed 


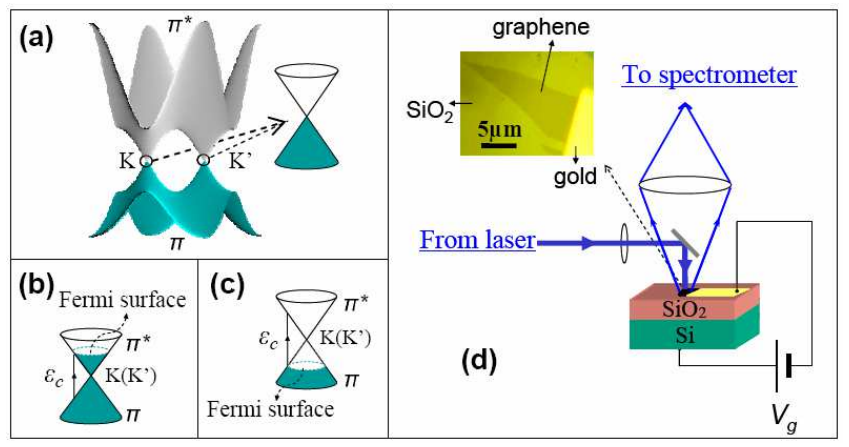

FIG. 1: (color online). Electronic band structure of graphene (a-c) and experimental set-up (d). (a) $\pi$ and $\pi^{*}$ bands of graphene in momentum space. $\mathrm{K}$ and $\mathrm{K}^{\prime}$ (Dirac points) are the corner points of the first Brillouin zone. For neutral graphene, the $\pi$ band is completely filled shown as blue shading in this diagram. Near Dirac points, the band structure exhibits isotropic linear dispersion shown as two cones at $\mathrm{K}$ and $\mathrm{K}$ '. (b) shows the region near Dirac points in n-type graphene. $\varepsilon_{c}$ is the onset energy for vertical electron-hole pair transitions. (c) is like (b) for p-type graphene. (d) Upper left is an optical image of the sample.

in a backscattering configuration (Fig. 1(d)) with the 488-nm line of an argon-ion laser as exciting radiation. The laser spot size on the sample was $\sim 25 \mu \mathrm{m}$ in diameter and the power was kept below $5 \mathrm{~mW}$. The scattered light was collected into a Spex-1404 0.85-m double grating spectrometer and spectra were recorded by a liquid nitrogen cooled CCD camera. The spectral resolution in these measurements is $\sim 2 \mathrm{~cm}^{-1}$.

Typical spectra showing $\mathrm{G}$ and $\mathrm{D}^{*}$ bands at cryostat cold finger temperatures $\sim 10 \mathrm{~K}$ are displayed in Fig. 2 , Remarkably, both peak position and line-width are found to be modulated by the gate voltage. The changes induced by the EFE are much larger for the $\mathrm{G}$ mode than for the second-order $\mathrm{D}^{*}$ band. We also show for comparison a $\mathrm{G}$ band for thick (>10 nm) samples (Fig. 2(b)) taken under similar experimental conditions with no gate voltage applied, which serves as a bulk sample spectrum.

The EFE induced charge in the single layer of graphene can be modulated by gate voltage according to the relation $n=C_{g}\left(V_{g}-V_{\text {Dirac }}\right) / e$, where $C_{g}$, e, and $V_{\text {Dirac }}$ are the gate capacitance, electron charge and the gate voltage corresponding to the charge-neutral Dirac-point. The changes in graphene Raman spectra as a function of $V_{g}$ demonstrate that the EFE modulated charge density results in significant changes in the frequency and lifetime of phonons.

Figure 3 displays the G-mode frequency $\omega_{G}$ and width $\Gamma_{G}$ as a function of $V_{g}$. It is remarkable that $\omega_{G}\left(V_{g}\right)$ and $\Gamma_{G}\left(V_{g}\right)$ both exhibit nearly symmetric changes relative to the value of $V_{g} \sim 18 \mathrm{~V}$. This symmetry is naturally linked to the symmetry of the electronic band structure that occurs at the Dirac point shown in Fig. 1(a), from which we determine $V_{\text {Dirac }}=18 \pm 2 \mathrm{~V}$. In particular, $\omega_{G}\left(V_{g}\right)$ has

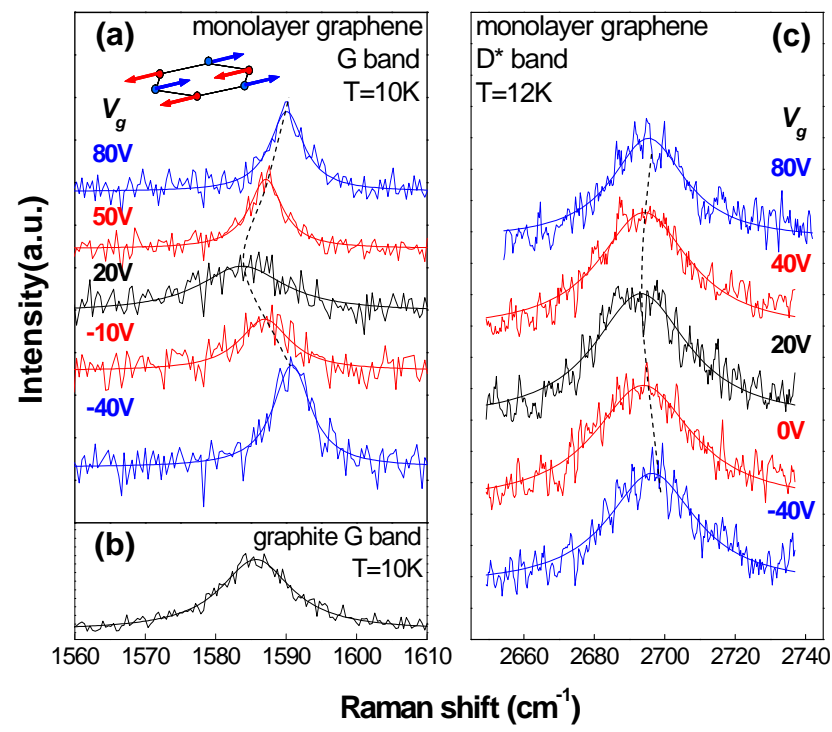

FIG. 2: (color online). Low temperature Raman spectra and their Lorentzian fits (smooth overlapping curves). (a) Evolution of the $\mathrm{G}$ band of a graphene monolayer with gate voltage $V_{g}$. The upper left inset is a schematic representation of carbon atom motion in the $\mathrm{G}$ band. The dashed line is a guide to the eye. (b) Spectrum of the $\mathrm{G}$ band of a thick $(>10 \mathrm{~nm}$ ) graphite layer. (c) Evolution of the $D^{*}$ two-phonon band of a graphene monolayer with $V_{g}$.

a minimum near the Dirac point, revealing that increases in charge density of either sign result in stiffening of the $G$ mode. On the other hand, $\Gamma_{G}$ sharply decreases as $\mid V_{g}-$ $V_{\text {Dirac }} \mid$ increases, showing that longer phonon lifetimes are linked to higher particle/hole density. These results offer key insights into the coupling of Dirac fermions to lattice vibration modes, as discussed below.

We first consider $\Gamma_{G}$ shown in Fig. 廿4(a). Its marked reduction (from 15 to $6.5 \mathrm{~cm}^{-1}$ ) at higher carrier densities can be understood as a Landau damping of phonons in which the mode decays into particle-hole pairs. Examples of such processes are shown in the inset to Fig. 4(a) and in Fig. 4(b). Phonon decay processes are real transitions that conserve energy and momentum. The small wavevector $\mathrm{G}$ phonon can only decay into particlehole pairs represented by vertical transitions that have vanishingly small wavevector transfer (Fig. 4(b)). When charge carriers are induced by EFE in ideal graphene at low temperatures, the Fermi level $E_{F}$ changes as $E_{F}(n)=-\operatorname{sgn}(n) \hbar v_{F} \sqrt{(\pi|n|)}$, where $v_{F}=10^{6} \mathrm{~m} / \mathrm{sec}$ [12, 13] is the Fermi velocity and $n$ is charge density. To satisfy the Pauli principle, Landau damping is allowed only when $\left|E_{F}\right|<\hbar \omega_{G} / 2$ (Figs. 4(b) and 4(c)). This is the relatively low density regime in which $\mathrm{G}$ band phonons are damped via decay into vertical particle-hole pairs.

The total change in $\Gamma_{G}$ is written as $\Delta \Gamma_{G}=$ $\Gamma_{G}\left(V_{\text {Dirac }}\right)-\Gamma_{G}^{0}$, where $\Gamma_{G}^{0}$ is the residual line-width from processes that are not related to Landau damping. The 


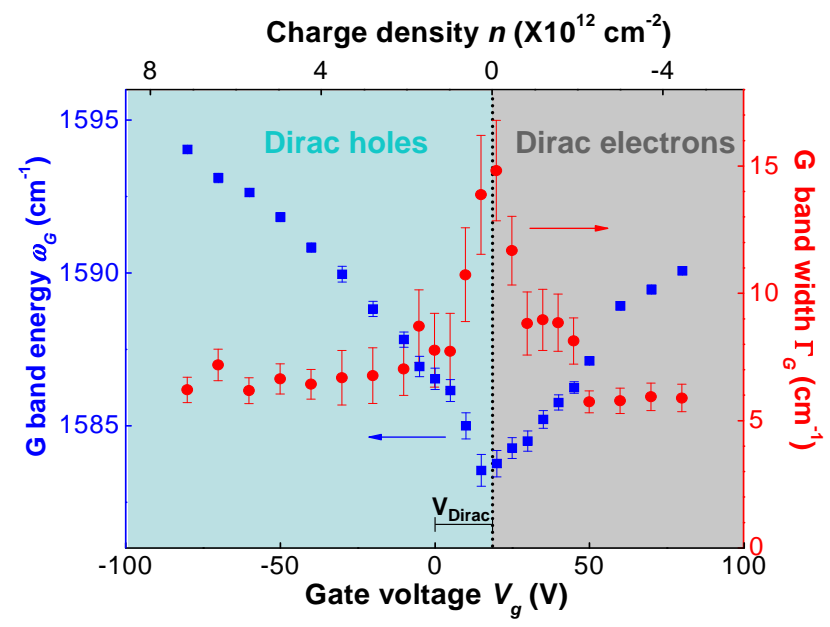

FIG. 3: (color online). G band energy (squares) and G band width (circles) extracted from Fig. 2(a). The vertical dotted line is the approximate position of the charge neutral Dirac point that is estimated from the symmetry of the data. The upper scale is obtained with $n=C_{g}\left(V_{g}-V_{\text {Dirac }}\right) / e$, where $C_{g}=115 \mathrm{aF} / \mu m^{2}$ [12, 13].

Landau damping phonon decay rate $\Delta \Gamma_{G}$ is the probability of creating energetically allowed electron-hole pairs via the electron-phonon coupling in unit time. Therefore, the electron-phonon coupling strength can be estimated from $\triangle \Gamma_{G}$. A calculation based on the Fermi golden rule yields [9]:

$$
\triangle \Gamma_{G}=\frac{A_{u c}}{8 M v_{F}^{2}} D^{2}
$$

where $A_{u c}$ is the area of the graphene unit cell, $M$ is carbon atom mass, and $D$ is the electron-phonon coupling strength. The measured $8.5 \mathrm{~cm}^{-1} \triangle \Gamma_{G}$ yields $D=14.1 \mathrm{eV} / \AA$, which seems to be consistent with the value predicted by density functional theory [21].

The results in Fig. 4(a) exhibit that the measured $\Gamma_{G}$ has departures from the step-like function (blue dashed line) expected for ideal graphene with uniform charge density distribution. In graphene samples, however, there are presumably local density variations due to selfdoping [22], chemical adsorbants [15] and charged impurities trapped in the substrate 23]. Different locations of the graphene sheet have thus different local contributions to damping of $\mathrm{G}$ phonons, smoothing out the sudden changes at $\pm \hbar \omega_{G} / 2$. Considering a non-uniform spatial variation of $\sim \pm 3 \times 10^{11} \mathrm{~cm}^{-2}$ in charge density, we obtained a reasonable fit for $\Gamma_{G}$ shown as the red solid curve in Fig. 4(a).

The EFE modulation of $\omega_{G}$, that has a minimum near the Dirac point, is shown in Fig. 4(d) as a function of Fermi energy. For carrier densities above $\sim \pm 5 \times 10^{11}$ $\mathrm{cm}^{-2}$ where non-uniformity is no longer dominant, the G-band phonon energy increases linearly with changes in Fermi energy. This intriguing result directly links the

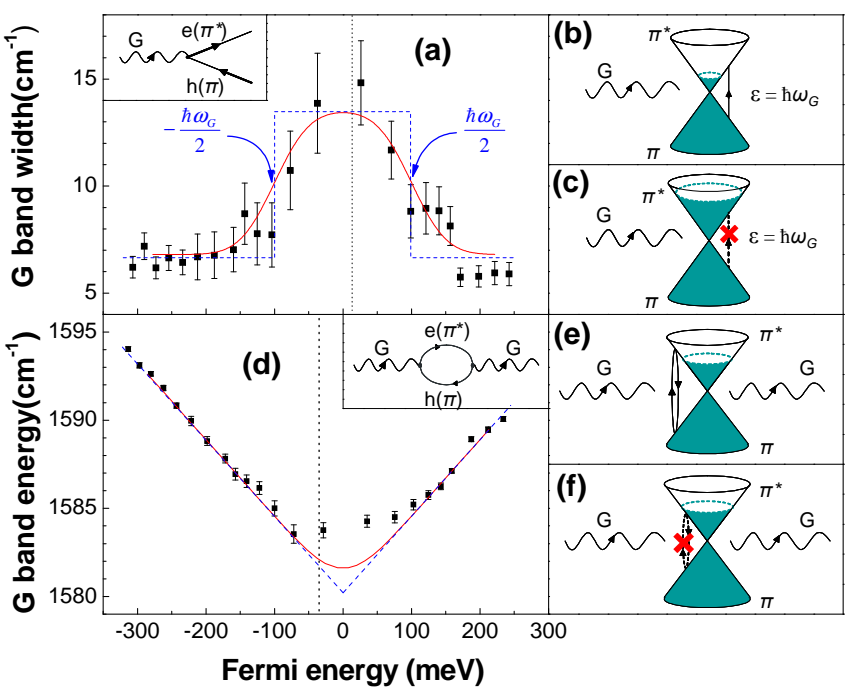

FIG. 4: (color online). Graphene G band damping (a-c) and energy renormalization (d-f). In (a) and (d), blue dashed lines and red solid lines are the fits for ideal and non-uniform graphene respectively. In all cases, the same electron-phonon coupling strength $(D=12.6 \mathrm{eV} / \AA)$ is assumed. The vertical dotted lines are the determinations of the Dirac point shown in Fig. 3. The insets are Feynman diagrams for electronphonon coupling applicable to the case of the $G$ phonon. (b) represents the broadening of the $\mathrm{G}$ phonon due to decay into particle-hole pairs. (c) indicates that the $G$ phonon decay into electron-hole pair is forbidden by the Pauli principle at high EFE induced charge densities. (e) is for the renormalization of the $G$ phonon energy by creation of virtual electron-hole pairs. (f) shows that virtual electron-hole pair transitions with energy ranging from 0 to $2\left|E_{F}\right|$ are forbidden. Only the diagrams for n-type graphene are shown in panels (b), (c), (e), and (f). Diagrams for the p-type cases are similar.

EFE-modulated $\omega_{G}$ to $2 \mathrm{D}$ Dirac fermion properties, as discussed below.

The coupling between $\mathrm{G}$ phonon lattice vibrations and Dirac fermions is allowed by graphene lattice symmetry. The carriers residing in the honeycomb lattice respond to the dynamical perturbation of $\mathrm{G}$ mode lattice vibration by creation and annihilation of virtual long-wavelength electron-hole pairs across the gapless Dirac point, which in turn, renormalizes the $\mathrm{G}$ phonon energy. The energy range of the virtual electron-hole pairs allowed by Pauli principle is decided by the position of Fermi level (Figs. 廿(e) and 4(f)). When the graphene is charge neutral, the onset energy is zero. If graphene is doped with electrons or holes, the onset energy is twice the Fermi energy. The difference of the $G$ band energy in neutral and charged graphene is thus given by the renormalization of electronhole pairs with energy ranging from 0 to $2\left|E_{F}\right|$.

Carrying out the calculation explicitly with time dependent perturbation theory 24] and the linear dispersion near the Dirac point, we find that the change of 
G-band phonon energy is described by

$$
\hbar \omega_{G}-\hbar \omega_{G}^{0}=\lambda\left\{\left|E_{F}\right|+\frac{\hbar \omega_{G}}{4} \ln \left|\frac{2\left|E_{F}\right|-\hbar \omega_{G}}{2\left|E_{F}\right|+\hbar \omega_{G}}\right|\right\}
$$

where $\omega_{G}^{0}$ is $\omega_{G}$ at Dirac point, $\lambda=\frac{A_{u c} D^{2}}{2 \pi \hbar \omega_{G} M v_{F}^{2}}$ [25]. In the high density limit, the linear term dominates over the logarithm term. A fit of the linear segments in Fig. 4(d) (blue dashed lines) with the linear term in Eq. (2) gives $D=12.6 \mathrm{eV} / \AA$, close to the value $14.1 \mathrm{eV} / \AA$ determined above from the analysis of the $\omega_{G}$ data. The red smooth line in Fig. $4(d)$ is the fit that takes into account the impact of non-uniformity described above.

It is worth noting that $\frac{\Delta \Gamma_{G}}{\lambda}=\frac{\pi \hbar \omega_{G}}{4} \approx 154 \mathrm{meV}$, which depends only on one parameter. Our experimental result gives $195 \mathrm{meV}$.

Finally, we consider the impact of the EFE on the D* Raman mode shown in Fig. 2(c). Although both peak position and width of the $\mathrm{G}$ and $\mathrm{D}^{*}$ bands exhibit similar gate dependence behavior, the magnitudes of the changes seen in Fig. 2(c) are only $\sim 10 \%$ of those of the G band. This smaller gate response can be explained by the impact of electron-phonon interactions for the two large wavevector phonons that contribute to the second-order Raman $\mathrm{D}^{*}$ band. The renormalization of the phonon frequency due to electron-phonon interactions is inversely proportional to the energy of particle-hole transitions at the phonon wavevector [24]. In this context, the weaker response of the $\mathrm{D}^{*}$ band to the EFE suggests that relevant EFE-modulated particle-hole transitions here have energies about an order of magnitude larger than those in the renormalization of the G band [26]. Further quantitative description could be carried out by the analysis of the doubly-resonant Raman processes in the $\mathrm{D}^{*}$ band [14].

In conclusion, we observed EFE-tunable electronphonon coupling in Raman spectra of single layer graphene. The gate voltage dependences of phonon frequency and damping reveal charge-tunable interactions of optical phonons with Dirac fermion transitions across a vanishing bandgap. These results uncover physics that links the EFE with electron-phonon coupling and demonstrate venues to probe fundamental interactions and to characterize atomic scale structures and devices.

Additional Remark: During the preparation of this manuscript, we became aware of related work on similar systems from other groups [27, 28, 29, 30].

We thank I. L. Aleiner, M. S. Hybertsen, A. J. Millis, S. Reich, J. Lin, J. Maultzsch, Y. Ahmadian and D. Basko for stimulating discussions. We acknowledge financial support from Nanoscale Science and Engineering Initiative of the National Science Foundation under NSF Award Number CHE-0117752 and CHE-0641523, the New York State Office of Science, Technology, and Academic Research (NYSTAR), and the Office of Naval
Research under Award Number N000140610138. P. K. acknowledges the support from FENA MARCO Center. A. P. is supported by the National Science Foundation under Award Number DMR-0352738, by the Department of Energy under Award Number DE-AIO2-04ER46133, and by a research grant of the W. M. Keck Foundation.

[1] S. Y. Zhou, G.-H. Gweon, and A. Lanzara, Annals of Physics 321, 1730 (2006).

[2] A. Bostwick et al., cond-mat/0609660 (2006).

[3] Z. Yao, C. L. Kane, and C. Dekker, Phys. Rev. Lett. 84, 2941 (2000).

[4] A. Javey et al., Phys. Rev. Lett. 92, 106804 (2004).

[5] J. Y. Park et al., Nano Lett. 4, 517 (2004).

[6] H. Suzuura and T. Ando, Phys. Rev. B 65, 235412 (2002).

[7] C. L. Kane and E. J. Mele, Phys. Rev. Lett. 78, 1932 (1997).

[8] S. Piscanec et al., Phys. Rev. Lett. 93, 185503 (2004).

[9] M. Lazzeri et al., Phys. Rev. B 73, 155426 (2006).

[10] M. S. Dresselhaus and P. C. Eklund, Adv. Phys. 49, 705 (2000).

[11] O. Dubay, G. Kresse, and H. Kuzmany, Phys. Rev. Lett. 88, 235506 (2002).

[12] K. S. Novoselov et al., Nature 438, 197 (2005).

[13] Y. Zhang, Y.-W. Tan, H. L. Stormer, and P. Kim, Nature 438, 201 (2005).

[14] S. Reich and C. Thomsen, Phil. Trans. R. Soc. Lond. A 362, 2271 (2004).

[15] K. S. Novoselov et al., Science 306, 666 (2004).

[16] C. Berger et al., Science 312, 1191 (2006).

[17] A. C. Ferrari et al., Phys. Rev. Lett. 97, 187401 (2006).

[18] A. Gupta et al., cond-mat/0606593 (2006).

[19] D. Grag et al., cond-mat/0607562 (2006).

[20] K. S. Novoselov et al., Proc. Natl. Acad. Sci. U.S.A. 102, 10451 (2005).

[21] Comparing Eq. (1) with Eq. (3) in Ref. [9], $\left\langle D_{\Gamma}^{2}\right\rangle_{F}=$ $\frac{D^{2}}{4}$. Ref. [9] claims that density functional theory gives $\left\langle D_{\Gamma}^{2}\right\rangle_{F}=45.60(\mathrm{eV} / \AA)^{2}$.

[22] N. M. R. Peres, F. Guinea, and A. H. Castro Neto, Phys. Rev. B 73, 125411 (2006).

[23] Y.-W. Tan, Y. Zhang, H. L. Stormer, and P. Kim (unpublished).

[24] W. E. Pickett and P. B. Allen, Phys. Rev. B 16, 3127 (1977).

[25] The 2nd term of Eq. (2) predicts a logarithmic divergence as $\left|E_{F}\right|$ approaches $\hbar \omega_{G} / 2$. In the experimental data, there is no obvious indication of such a divergence, most likely because it is smoothed out by broadening due to non-uniformity.

[26] The strength of electron-phonon coupling is similar for $\mathrm{G}$ and D* phonons. See Eq. (11) of Ref. [8].

[27] A. H. Castro Neto and F. Guinea, cond-mat/0608543 (2006).

[28] T.Ando, J. Phys. Soc. Jpn. 75, 124701 (2006).

[29] M. Lazzeri and F. Mauri, Phys. Rev. Lett. (to be published).

[30] S. Pisana et al., cond-mat/0611714 (2006). 